\title{
Cocultivation of Mesenchymal Stem Cells and Endothelial Progenitor Cells Reveals Antiapoptotic and Proangiogenic Effects
}

\author{
Dominik Steiner $^{\mathrm{a}}$ Katrin Köhn ${ }^{\mathrm{a}} \quad$ Justus P. Beier ${ }^{\mathrm{a}}$ Michael Stürzl $^{\mathrm{b}}$ \\ Raymund E. Horch ${ }^{\mathrm{a}}$ Andreas Arkudas ${ }^{\mathrm{a}}$ \\ ${ }^{a}$ Department of Plastic and Hand Surgery, and ${ }^{b}$ Division of Molecular and Experimental Surgery, University Hospital \\ of Erlangen, Friedrich-Alexander University of Erlangen-Nürnberg (FAU), Erlangen, Germany
}

\section{Keywords}

Stem cells - Regenerative medicine - Proliferation . Apoptosis · Angiogenesis

\begin{abstract}
Integrating bioartificial tissues into the host vasculature is a prerequisite for tissue engineering applications. Endothelial progenitor cells (EPCs) display a high angiogenic potential and a low donor-site morbidity, making them ideal for tissue engineering applications. In our study we used a murine EPC cell line (T17b) and rat mesenchymal stem cells (MSCs) for cocultivation experiments. MSCs were cocultured with increasing T17b EPC amounts. Furthermore, MSCs in monoculture were treated with conditioned medium (CM) from T17b EPCs and T17b EPCs were treated with CM from MSCs. Proliferation and apoptosis were quantified with a bromodeoxyuridine ELISA and a DNA fragmentation ELISA, respectively. Osteogenic differentiation was detected with an alkaline phosphatase assay and bone morphogenetic protein-2 ELISA. The production of proangiogenic molecules was measured with a matrix metalloproteinase-3 and vascular endothelial growth factor ELISA as well as nitric oxide assay. We
\end{abstract}

\section{KARGER}

(C) 2017 S. Karger AG, Basel

E-Mail karger@karger.com

www.karger.com/cto could show that T17b EPCs stimulated MSC proliferation but not vice versa. On the other hand, MSCs promoted the cell survival of EPCs. The growth-inducing and antiapoptotic effects were dependent on heterotypic cell contacts and paracrine mediators. Moreover, proangiogenic growth factors were found in the coculture. Collectively, our results indicate that the coapplication of MSCs and T17b EPCs provides new perspectives for tissue engineering applications.

(c) 2017 S. Karger AG, Basel

\section{Abbreviations used in this paper}

ALP alkaline phosphatase

BMP bone morphogenetic protein

BrdUrd bromodeoxyuridine

CM conditioned medium

DM differentiation medium

EPCs endothelial progenitor cells

MMP matrix metalloproteinase

MSCs mesenchymal stem cells

NO nitric oxide

PBS phosphate-buffered saline

VEGF vascular endothelial growth factor 


\section{Introduction}

Despite the advantages in microsurgery, the reconstruction of large-volume tissue defects as a result of trauma or cancer still represents a therapeutic challenge for the plastic surgeon. Autologous tissue transfer is the current gold standard for the treatment of such defects. On the other hand, autologous tissue transfer is limited by the defect size and donor site morbidity. A promising approach to overcoming these problems is the emerging field of tissue engineering. However, adequate vascularization of tissue-engineered constructs is the crucial step for clinical implementation. In the initial phase of the implantation of bioartificial tissues into the host vasculature, oxygen and nutrition supply depends on diffusion. As diffusion is limited to a maximum range of 100-200 $\mu \mathrm{m}$, various strategies have been developed to improve vascularization [Ko et al., 2007; Rouwkema and Khademhosseini, 2016]. These therapeutic approaches comprise the delivery of angiogenic growth factors [Street et al., 2002; Arkudas et al., 2007; Moimas et al., 2015], the implementation of endothelial cells [Koob et al., 2011], or the microsurgical improvement of the vascularization by the use of arteriovenous loops [Horch et al., 2014; Weigand et al., 2015]. As endothelial progenitor cells (EPCs) display a high angiogenic and vasculogenic potential as well as a low donor site morbidity they represent an interesting cell source for the prevascularization of bioartificial tissues [Asahara et al., 1997; Kupatt et al., 2005b; Yoder et al., 2007; Young et al., 2007]. It is generally accepted that EPCs are recruited from the bone marrow to hypoxic tissues or tumor parenchyma supporting neovascularization [Asahara et al., 1999; Ahn and Brown, 2009]. Based on these findings first efforts have been made to use the angiogenic and vasculogenic potential of EPCs in a therapeutic manner [Folkman, 1998; Kocher et al., 2001; Iba et al., 2002; Kupatt et al., 2005a; Wang et al., 2015]. For the recent study, we used the well-established EPC cell line T17b isolated from mice embryos on day E7.5 [Hatzopoulos et al., 1998]. Endothelial differentiation of T17b EPCs can easily be induced in vitro with retinoic acid and cAMP as previously shown by an upregulation of endothelial marker genes such as thrombomodulin, flk-1, or von Willebrand factor [Hatzopoulos et al., 1998; Bleiziffer et al., 2009]. Moreover, T17b EPCs assume the characteristic cobblestone morphology and form tube-like structures when seeded onto Matrigel as a proof of their endothelial character [Hatzopoulos et al., 1998]. In a previous study we could demonstrate that T17b EPCs proliferate, form tube-like structures, and se-

Antiapoptotic and Proangiogenic Effects

of Cocultivation of MSCs and EPCs crete vascular endothelial growth factor (VEGF) in vitro when seeded into a fibrin matrix [Bleiziffer et al., 2009]. Furthermore, we could demonstrate that T17b EPCs display an increased proliferation rate as well as VEGF-secretion under hypoxic conditions which might be an explanation for their great vasculogenic potential, especially in ischemic tissues [Asahara et al., 1999]. As a next step, we subcutaneously implanted T17b EPCs in a fibrin matrix and found that T17b EPCs support the formation of fibrovascular tissue as indicated by an increased blood vessel quantity and diameter [Bleiziffer et al., 2011].

Mesenchymal stem cells (MSCs) have also gained much interest in the field of regenerative medicine because of their capability of differentiating into multiple cell types, including adipocytes, osteoblasts, chondrocytes, and even endothelial cells [Janeczek Portalska et al., 2012].

T17b EPCs do not express major histocompatibility complex I molecules and therefore can be used as an interesting cell source for xenogenic cell transplantation. Prior a possible in vivo application, we performed cocultures with rat MSCs and T17b EPCs and investigated whether coculturing has supportive effects on proliferation, apoptosis, osteogenic differentiation, and angiogenesis in vitro. Furthermore, we tried to figure out the ideal cell ratio of MSCs and T17b EPCs in order to improve the generation of bioartificial tissues based on both cell types.

\section{Materials and Methods}

\section{Cell Culture}

MSC Isolation and Cultivation

The isolation of MSCs was approved by the Animal Care Committee of the University of Erlangen and the Government of Mittelfranken, Germany (55.2-2532.1-53/14). MSCs were isolated and characterized by an established protocol [Rottensteiner et al., 2014; Vielreicher et al., 2015]. Briefly, male Lewis rats were sacrificed, the femoral bones were prepared, and the bone marrow was flushed with phosphate-buffered saline (PBS) and fetal calf serum (Biochrom, Berlin, Germany). The cell suspension was centrifuged for $10 \mathrm{~min}$ at 15,000 rpm and the cell pellet was resolved in DMEM (Gibco/Life Technologies, Carlsbad, CA, USA) containing 20\% fetal bovine serum (Biochrom), 1\% penicillin/streptomycin (Gibco Invitrogen) and 1\% L-glutamine (Sigma-Aldrich, Schnelldorf, Germany). The cells were filtered through $100-\mu \mathrm{m}$ cell strainers (BD ${ }^{\mathrm{TM}}$, Becton Dickinson, Heidelberg, Germany) and purified by density gradient centrifugation with Histopaque ${ }^{\circledR}$ (Sigma-Aldrich). Thereafter, the cells were seeded in culture flasks at a density of $2.0 \times 10^{6} / \mathrm{cm}^{2}$ in a humidified atmosphere $\left(37^{\circ} \mathrm{C} ; 5 \% \mathrm{CO}_{2}\right)$. After $48 \mathrm{~h}$, the medium was changed and nonadherent cells were washed out. The remaining cells were further cultured until they reached a confluence of $80 \%$. Then, cells were detached from the cell culture dishes with Accutase solution (PromoCell, Heidelberg, Germany) and used until passage 5.

Cells Tissues Organs 2017;204:218-227

DOI: $10.1159 / 000478654$ 


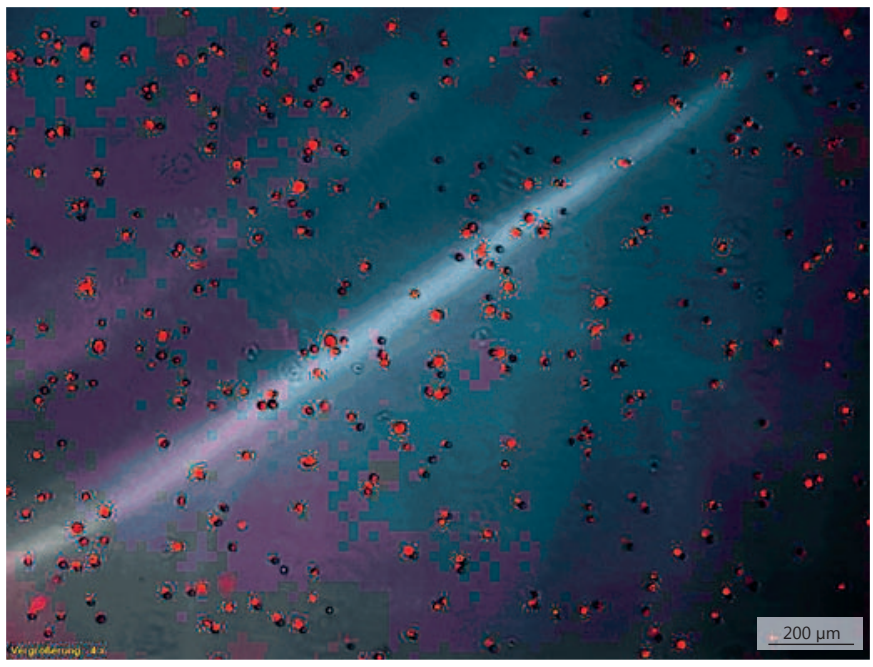

Fig. 1. After $72 \mathrm{~h}$ of incubation in DM, T17b EPCs stained positive for DiI-ac-LDL.

T17b EPC Cultivation and Differentiation

Cell culture flasks (Greiner Bio-One, Frickenhausen, Germany) were coated with bovine skin gelatin type B (Sigma-Aldrich, Schnelldorf, Germany) prior to seeding with T17b EPCs. Cell cultivation was performed using a basal medium (BM) containing high glucose DMEM GlutaMAX ${ }^{\circledR}$ (Gibco/Life Technologies), 20\% fetal calf serum (Biochrom), $100 \mathrm{U} / \mathrm{mL}$ penicillin (Biochrom), 100 $\mu \mathrm{g} / \mathrm{mL}$ streptomycin (Biochrom), $1 \mathrm{~mm}$ nonessential amino acids (Gibco), 2 mM HEPES buffer pH 7.5 (Gibco), and 0.1 mM 2-mercaptoethanol (Gibco). Endothelial differentiation was induced by supplementing $1 \mu \mathrm{M}$ all-trans retinoic acid (Sigma-Aldrich) and $0.5 \mathrm{~mm}$ dibutyryl cyclic AMP (Sigma-Aldrich) for $72 \mathrm{~h}$, as described by Hatzopoulos et al. [1998]. Endothelial differentiation was confirmed by positive DiI-ac-LDL staining as previously described (Fig. 1) [Brandl et al., 2014].

Experimental Design

In order to differentiate between the effects due to heterotypic cell contacts or paracrinely acting mediators, 2 dimensional cocultures of MSCs and T17b EPCs and monocultures of MSCs or T17b EPCs were performed. To determine the optimal T17b EPC/MSC cell ratio, MSCs were cocultured with 25, 50, and 75\% T17b EPCs. Monocultures of MSCs received differentiation medium (DM) or conditioned medium (CM) from T17b EPCs. Vice versa, monocultures of T17b EPCs received DM or CM gained from MSCs. $\mathrm{DM}$ was used for mono- and cocultivation experiments. $\mathrm{CM}$ was gained from supernatants of T17b EPC or MSC monocultures after $48 \mathrm{~h}$, and supplemented with fresh DM in a 1:1 ratio. We used $1.3 \times 10^{4}$ cells $/ \mathrm{cm}^{2}$ except in the bromodeoxyuridine (BrdUrd) ELISA $\left(5 \times 10^{3} / \mathrm{cm}^{2}\right)$.

\section{BrdUrd ELISA}

The effect of T17b EPCs on MSC cell proliferation was investigated using a BrdUrd cell proliferation ELISA. According to manufacturer's instructions (Roche Diagnostics, Mannheim, Germany), cell culture dishes were washed with PBS after each time point
$(24,72,120 \mathrm{~h})$ and BrdUrd labeling solution was added for $24 \mathrm{~h}$. Briefly, an anti-BrdUrd antibody labeled with peroxidase was added and subsequently substrate solution was added. Thereafter, absorbance was analyzed at $450 \mathrm{~nm}$ and a reference wavelength of $690 \mathrm{~nm}$ with an ELISA reader.

\section{DNA Fragmentation ELISA}

To quantify the rate of apoptotic cells a cell death detection ELISA (Roche Diagnostics, Mannheim, Germany) was carried out. Briefly, cells were detached from cell culture dishes after $72 \mathrm{~h}$. Firstly, the cells were centrifuged at 12,000 rpm for 5 min including the supernatants. Thereafter, cell lysis was performed. The resulting supernatants containing the fragmented DNA were transferred onto a microtiter plate coated with an antihistone antibody. Afterwards, a second anti-DNA antibody labeled with peroxidase and the ABTS (2,20-Azino-di[3-ethylbenzthiazolin-sulfonat]) substrate solution were added. Photometric analysis was performed with an ELISA reader at $405 \mathrm{~nm}$ and a reference wavelength of $490 \mathrm{~nm}$.

\section{VEGF ELISA}

VEGF production was measured using a mouse VEGF Quantikine ${ }^{\circledR}$ ELISA kit (R\&D Systems, Wiesbaden, Germany). Briefly, the supernatants of 3 samples per group were collected after $72 \mathrm{~h}$ and transferred to an anti-VEGF-coated microplate. VEGF was quantified by measuring the absorbance at $450 \mathrm{~nm}$ and reference wavelength of $540 \mathrm{~nm}$ with an ELISA reader.

\section{Matrix Metalloproteinase-3 ELISA}

Corresponding to manufacturer's instructions (R\&D Systems, Wiesbaden, Germany), a Quantikine ${ }^{\circledR}$ ELISA was carried out to quantify the production of matrix metalloproteinase (MMP)-3. Briefly, supernatants of 3 samples per group were gathered after $72 \mathrm{~h}$ and transferred to an anti-MMP-3-coated microplate. Thereafter, a second antibody specific for MMP-3 conjugated to horseradish peroxidase and the substrate solution were added. Absorbance was measured at $450 \mathrm{~nm}$ and a reference wavelength of 540 $\mathrm{nm}$ with an ELISA reader.

\section{Nitric Oxide Assay}

Nitric oxide (NO) assay was performed using the improved Griess method. Briefly, cells were detached from cell culture dishes and sonicated. After centrifugation (10,000 rpm), the supernatants were treated with $\mathrm{ZnSO} 4$ for deproteination. Colorimetric determination was performed following manufacturer specification (Antibodies-Online Inc., Atlanta, GA, USA). Absorbance was measured at $540 \mathrm{~nm}$ with an ELISA reader.

\section{Alkaline Phosphatase Assay}

The enzyme activity of the osteogenic differentiation marker alkaline phosphatase (ALP) was measured as described previously [Hager et al., 2009]. Briefly, cells were washed with PBS after $48 \mathrm{~h}$ of cell culturing. A lysis buffer was added containing $25 \mathrm{mM}$ of Tris- $\mathrm{HCl}(\mathrm{pH} 8.5)$ and $0.5 \%$ Triton X-100, and cell lysis was conducted by 3 freeze-thaw cycles. Thereafter, CSPD substrate (Applied Biosystems, Forster City, CA, USA) was added to the samples and the luminescence was measured. 


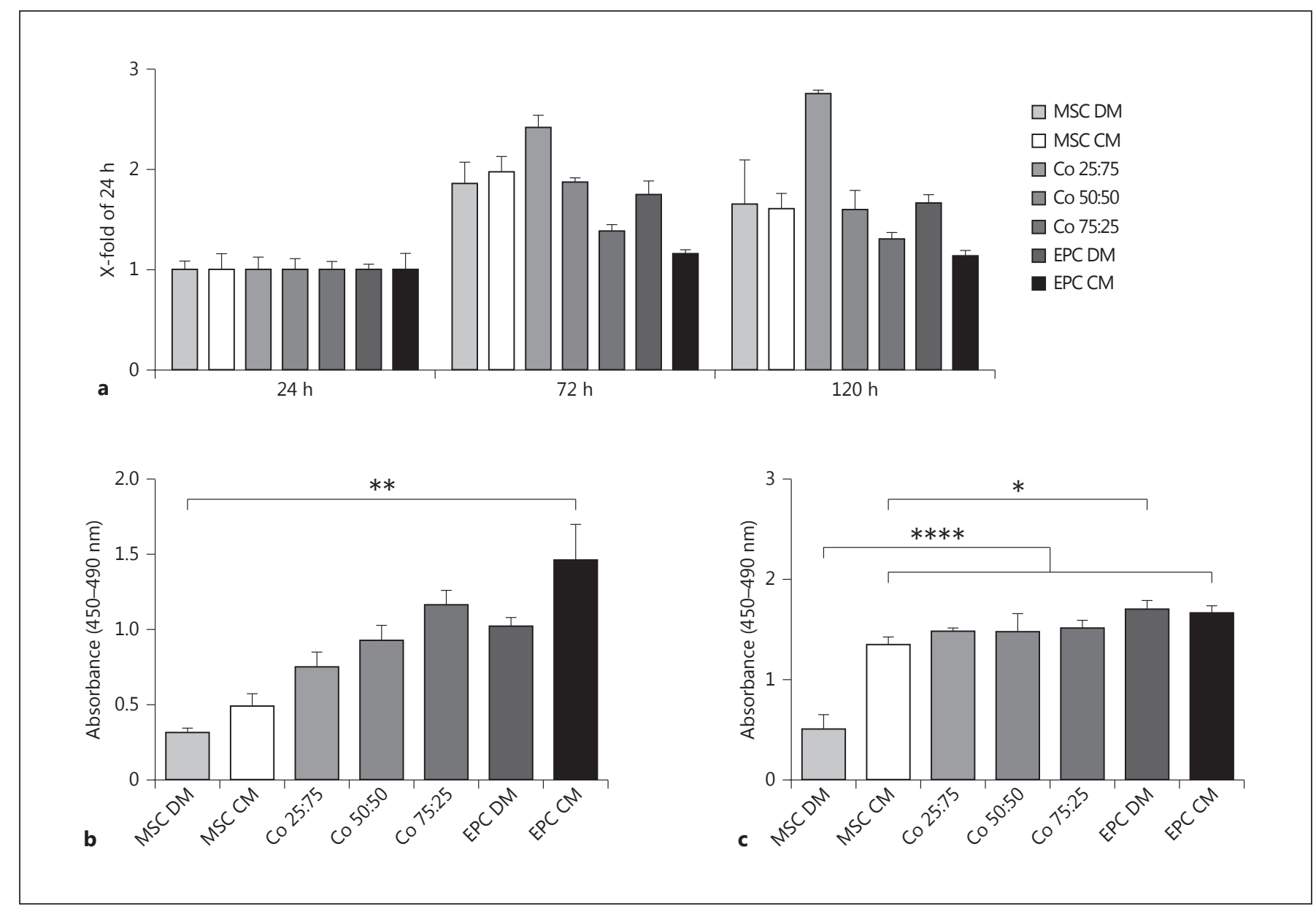

Fig. 2. Proliferation of T17b EPCs and MSCs under mono- and coculture conditions. a Proliferation was measured via BrdUrd incorporation and depicted as the relative increase compared to the BrdUrd amount $24 \mathrm{~h}$ after cell seeding. The subfigures (b, c) depict the absolute proliferation rate (shown as absorbance) 24 and $120 \mathrm{~h}$ after cell seeding. MSCs and T17b EPCs were grown under monoculture conditions with DM and served as the control groups. MSCs were treated with CM from T17b EPCs and T17b EPCs were treated with CM from MSCs. Cocultures were performed with an increasing T17b EPC cell ratio, beginning with 25\% up to $75 \%$ (Co 25:75, Co 50:50, Co75:25). Statistically significant differences are indicated as $* \leq 0.05,{ }^{* *} p \leq 0.01$, and $* * * * p<0.0001$.

\section{Bone Morphogenetic Protein-2 ELISA}

According to the manufacturer's instructions, an immunoassay kit (R\&D Systems, Wiesbaden, Germany) was used to quantify the bone morphogenetic protein (BMP)-2 production. Briefly, the supernatants were collected after $72 \mathrm{~h}$ of cell culturing and transferred onto a microplate coated with a BMP-2 antibody. Absorbance was measured at $450 \mathrm{~nm}$ and a reference wavelength of 540 $\mathrm{nm}$ with an ELISA reader.

\section{Statistical Analysis}

We first tested for a Gaussian distribution. If the data were normally distributed, a 1-way ANOVA and Bonferroni correction for multiple testing were performed. Otherwise, a nonparametric analysis was performed with the Kruskal-Wallis test and Dunn multiple comparisons test. $p$ values $\leq 0.05$ were considered statistically significant. Data are either shown as mean arbitrary units \pm
SD or box plots from triplicate determinations. GraphPad Prism 7.00 (GraphPad Software, San Diego, CA, USA) was used for the statistical analysis.

\section{Results}

\section{Effects of Coculturing T17b EPCs and MSCs on Proliferation}

In order to analyze the influence of coculturing T17b EPCs and MSCs on cell proliferation, a BrdUrd ELISA was performed. In general, the BrdUrd incorporation had increased in all groups in relation to the BrdUrd amount at $24 \mathrm{~h}$ after cell seeding (Fig. 2a). At that time point 


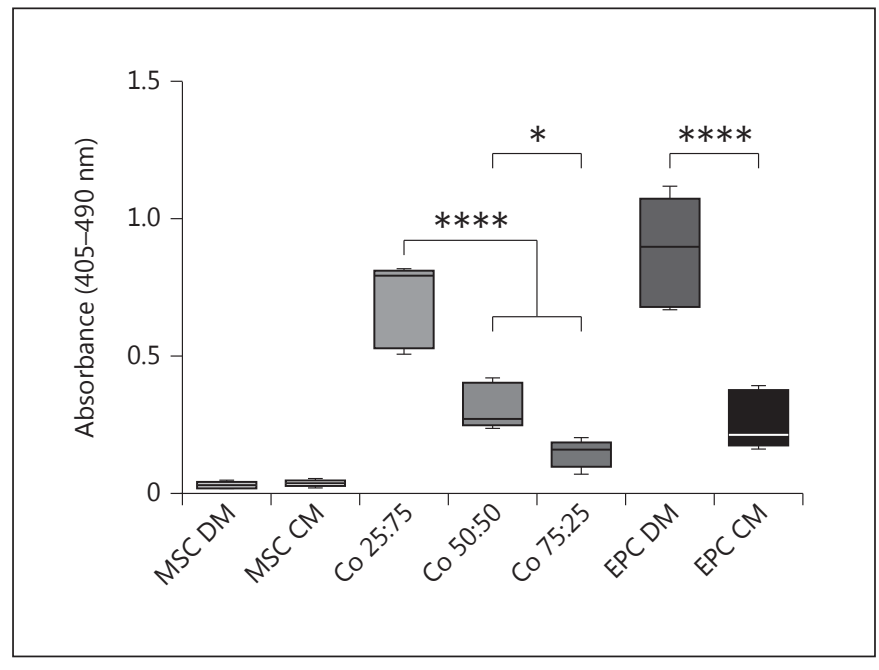

Fig. 3. Apoptosis of T17b EPCs and MSCs quantified by DNA fragmentation ELISA $72 \mathrm{~h}$ after cell seeding. MSCs and T17b EPCs were grown under monoculture conditions with DM and served as the control groups. MSCs (MSC CM) were treated with CM from T17b EPCs. T17b EPCs (T17b EPC CM) were treated with CM from MSCs. Cocultures were performed with an increasing T17b EPC cell ratio, beginning with 25\% up to 75\% (Co 25:75, Co 50:50, Co75:25). Statistically significant differences are indicated as $* p \leq 0.05$ and ${ }^{* * * *} p<0.0001$.

(Fig. 2b), T17b EPCs treated with DM or CM, displayed higher proliferation rates compared to the corresponding MSC cultures (MSC DM and MSC CM). Furthermore, there was a statistically significant difference between T17b EPCs treated with CM gained from MSCs and MSCs cultured in DM $(p<0.01)$. In the coculture groups, we could detect higher absolute proliferation rates with an increasing MSC cell ratio after $24 \mathrm{~h}$, although this trend was not statistically significant (Fig. 2b). Interestingly, MSCs displayed a statistically significant lower absolute proliferation rate under monoculture conditions compared to the other groups after $120 \mathrm{~h}(p<0.0001)$. Moreover, CM from T17b EPCs seemed to have a strong influence on MSC proliferation but not vice versa $(p<$ 0.0001; Fig. 2c).

\section{Effects of T17b EPC and MSC Coculture on Cell Survival}

A DNA-fragmentation ELISA was carried out to investigate whether MSCs have an effect on T17b EPC cell survival. For this reason, T17b EPCs and MSCs were treated with $\mathrm{CM}$ or grown under coculture conditions for 72 h. As shown in Figure 3, cocultures of MSCs and T17b EPCs displayed a lower apoptosis rate compared to the

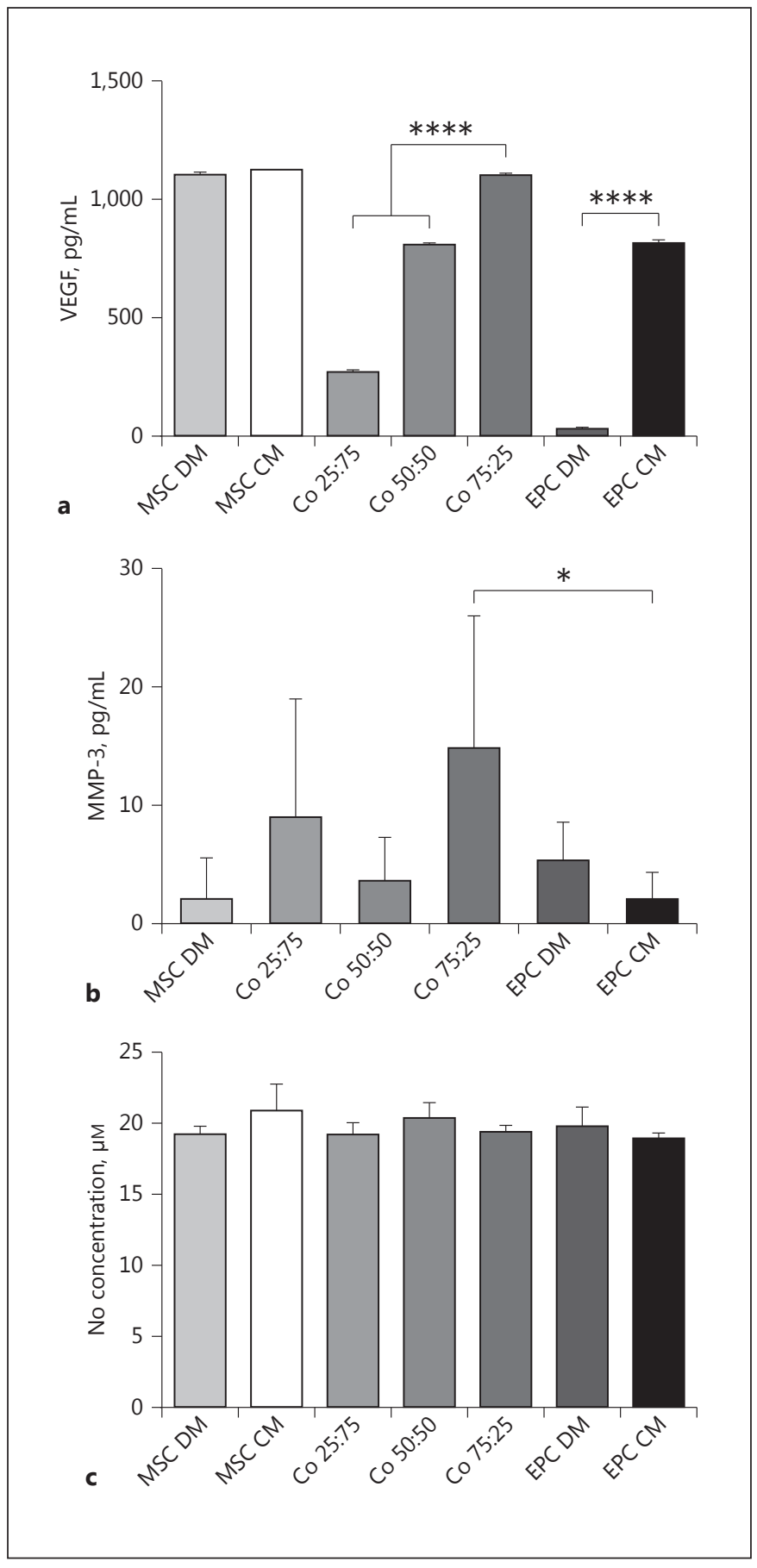

Fig. 4. Measurement of angiogenic factors produced by T17b EPCs and MSCs. Experiments were performed $72 \mathrm{~h}$ after cell seeding. MSCs and T17b EPCs were either grown under monoculture conditions with DM or treated with CM. Cocultures were performed with an increasing T17b EPC cell ratio, beginning with $25 \%$ up to 75\% (Co 25:75, Co 50:50, Co75:25). The concentration of VEGF (a) and MMP-3 (b) are indicated as pg/mL. c The NO concentration was measured using the Griess method and is indicated as $\mu \mathrm{M}$. Statistically significant differences are indicated as $* p \leq 0.05$ and $* * * * p<0.0001$. 


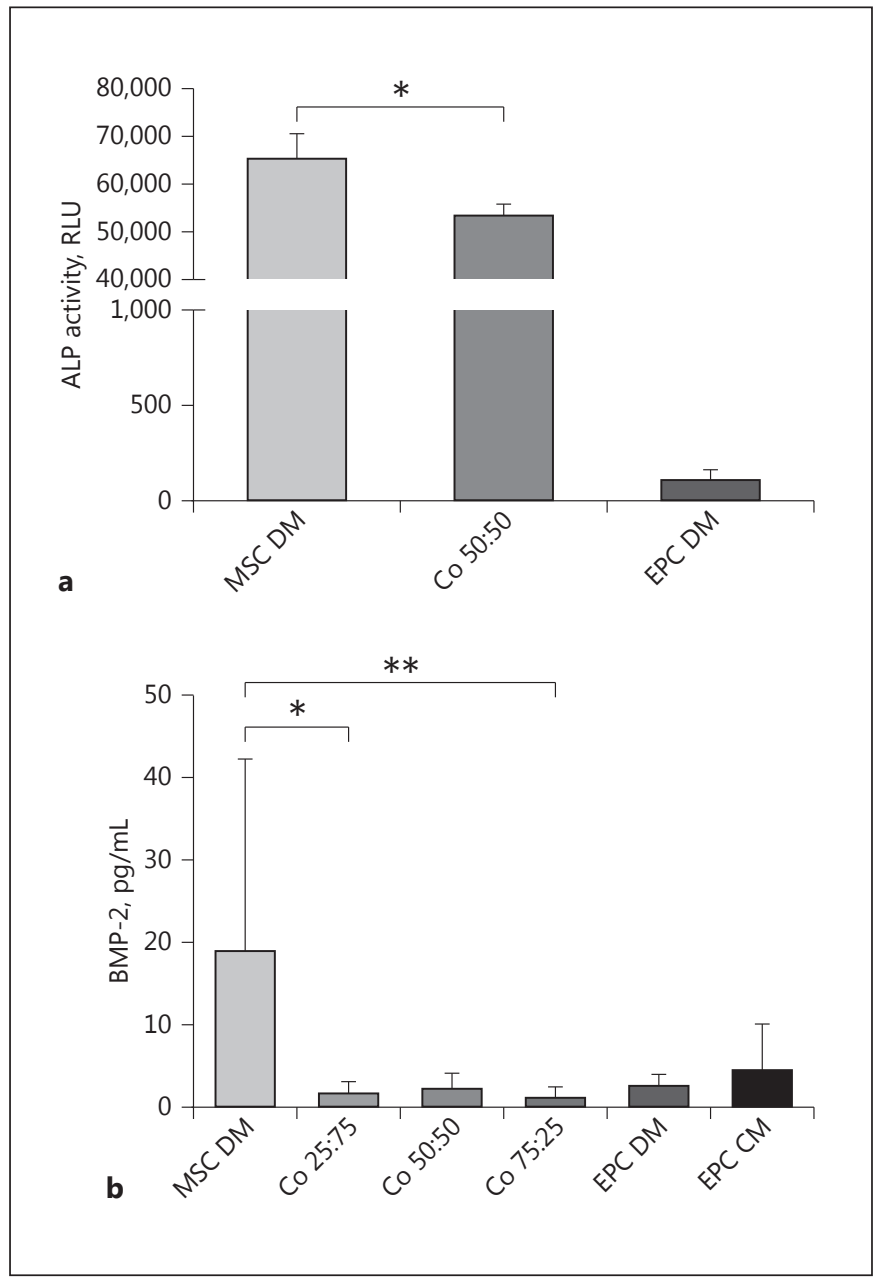

Fig. 5. Quantification of osteogenic differentiation markers of ALP in relative luminescence units (RLU) and BMP-2 (pg/mL). a For the ALP assay, MSCs were either grown in monoculture (MSC DM) or in coculture with T17b EPCs (Co 50:50) for 48 h. b For BMP-2 ELISA, MSCs were either grown under monoculture conditions (MSC DM) or cocultured with T17b EPCs with an increasing T17b EPC cell ratio, beginning with 25\% up to $75 \%$ (Co 25:75, Co 50:50, Co 75:25). The BMP-2 ELISA was performed after $72 \mathrm{~h}$. Statistically significant differences are indicated as $* p \leq 0.05$ and ** $p \leq 0.01$.

T17b EPC cultures (T17b EPC DM/CM). Interestingly, this effect was even more pronounced with an increasing MSC cell number in the coculture groups. By using CM from MSCs, the apoptosis rate could be significantly reduced in the T17b EPC monoculture group, whereas the apoptosis rate of MSCs treated with CM from T17b EPCs was unaffected, indicating that MSCs reduced the apoptosis of T17b EPCs through direct cell-cell contacts as well as paracrine-acting soluble mediators.

Antiapoptotic and Proangiogenic Effects of Cocultivation of MSCs and EPCs

\section{T17b EPCs and MSCs Produce and Secret}

\section{Proangiogenic Molecules}

As a next step, we investigated the production of factors involved in angiogenesis, namely VEGF, MMP-3, and NO. Under monoculture conditions, the amount of VEGF in MSCs was higher compared to T17b EPCs (Fig. 4a). The treatment with CM from T17b EPCs did not affect the VEGF production in MSCs. On the contrary, T17b EPCs treated with CM from MSCs displayed a higher VEGF amount compared to T17b EPCs treated with DM $(p<0.0001)$. Furthermore, we could detect a cell-dependent increase of VEGF in the coculture groups. It is well known that angiogenesis also involves the degradation of the extracellular matrix. By performing an MMP-3 ELISA, we could measure the highest MMP-3 amount in the coculture group containing $75 \%$ MSCs (Fig. 4b). Interestingly, we detected lower amounts of MMP-3 in the T17b EPC group treated with CM from MSCs compared to the untreated T17b EPCs. By using a $\mathrm{NO}$ assay, we could prove that all groups produced NO with no relevant differences between each treatment group (Fig. 4c).

\section{Influence of Coculturing T17b EPCs and MSCs on Osteogenic Differentiation}

In order to investigate the osteogenic differentiation, we performed an ALP assay as well as a BMP-2 ELISA. As shown in Figure 5, we could not prove a stimulation of the osteogenic differentiation by coculturing MSCs and T17b EPCs. Moreover, we could detect a statistically significant decrease of ALP activity in the coculture group $(p<0.05$; Fig. 5a). By measuring the production of BMP2 , we could also detect a decrease of this osteoinductive factor in all coculture groups, indicating that T17b EPCs do not stimulate the osteogenic differentiation of MSCs (Fig. 5b).

\section{Discussion}

The present study aimed at investigating the interplay between the murine EPC cell line T17b and rat MSCs concerning proliferation, apoptosis, osteogenic differentiation, and angiogenesis. A BrdUrd ELISA was performed to investigate the effect of coculturing MSCs and T17b EPCs on cell proliferation. Our results display a strong effect of T17b EPCs on MSCs proliferation, which is coherent with previous studies demonstrating that endothelial cells as well as EPCs have an impact on MSC proliferation [Steiner et al., 2012; Wen et al., 2016]. This

Cells Tissues Organs 2017;204:218-227 223 
effect seems to be stimulated by heterotypic cell-cell contacts as well as paracrine-acting mediators if CM was used. We could prove that the proliferation rate of MSCs was highly stimulated by paracrine-acting soluble mediators secreted from T17b EPCs. These results conform with other studies demonstrating that CM from endothelial cells or EPCs enhanced the proliferation of MSCs [Villars et al., 2000; Wen et al., 2016]. Conversely, CM from MSCs had no effect on T17b EPC proliferation. All coculture groups displayed higher proliferation rates compared to the MSCs in the monoculture after $72 \mathrm{~h}$, but we could not detect any differences between the coculture groups and the T17b EPCs. It is alluring to speculate that the growth-supporting effect of the T17b EPCs in the coculture system might be unidirectional and restricted to the MSCs.

Next, we investigated whether MSCs might have an effect on T17b EPC survival. Our data revealed that coculturing MSCs and T17b EPCs has an antiapoptotic effect in the 2-dimensional cell culture. This effect was even more pronounced with an increasing MSC amount in the coculture groups. In this context, gap junctions might play a role in intercellular signaling concerning cell survival in the direct cocultures [Villars et al., 2002; Hager et al., 2009]. Using CM from MSCs, we could prove that MSCs also promote cell survival of T17b EPCs in a paracrine manner. Moreover, we could demonstrate that cocultures of MSCs and T17b EPCs displayed a lower apoptosis rate compared to T17b EPCs cultured in DM (T17b EPC DM). As the apoptosis rate decreases with increasing MSC amounts in the cocultures, MSCs might increase the survival of T17b EPCs.

On the other hand, cell survival of MSCs seemed to be unaffected if CM from T17b EPCs was used. Interestingly, we could prove a relatively high apoptosis rate in the T17b EPC groups. Considering the results of a previous study using MSCs and HUVECs (human umbilical vein endothelial cells), it seems to be a normal phenomenon that endothelial cells display higher apoptosis rates compared to MSCs [Steiner et al., 2012]. Considering the fact that MSCs produced VEGF under mono- or coculture conditions, these antiapoptotic effects might be explained. In our experiments, we have observed an inverse correlation between increasing VEGF amounts and decreasing apoptosis in the coculture groups and the T17b EPCs treated with CM, indicating a strong effect of VEGF on T17b EPC cell survival. Our results are consistent with the pertinent literature, reporting that VEGF is an important survival factor for endothelial cells [Gerber et al., 1998a, b]. These authors demonstrated that the addition of
VEGF induced the expression of the antiapoptotic signal molecules Bcl-2 and A1 in HUVECs. Other cell types, such as osteoblasts, also secrete VEGF, thereby regulating the apoptosis by increased BCL-2 gene expression [Street and Lenehan, 2009]. In accordance with the work of Thébaud et al. [2012], we could observe a significant decline of VEGF in the coculture groups containing $\leq 50 \%$ MSCs compared to the MSC monoculture. On the other hand, if 75\% MSCs were used in the cocultures with T17b EPCs, we could detect an equal amount of VEGF compared to the MSC monoculture. Previous studies demonstrated that endothelial cells stimulate the gene expression of VEGF in cocultured human osteoprogenitor cells [Grellier et al., 2009; Kolbe et al., 2011]. In our own study, we could not prove that coculturing MSCs and T17b EPCs stimulated the VEGF protein secretion compared to the MSC monoculture, which is in accordance with the results of Kolbe et al. [2011]. However, it is important to bear in mind that the relative gene expression does not necessarily correlate with the level of protein expression. On the other hand, the VEGF production in the MSC monoculture could also be dependent on the selected cell culture medium [Kolbe et al., 2011]. Taken together, our experiments reveal that proliferation and apoptosis seem to be regulated via direct cell-cell interactions as well as paracrine mediators.

As previously demonstrated by Brandl et al. [2014], we could confirm endothelial differentiation of T17b EPCs by DiI-ac-LDL uptake. In order to prove that coculturing MSCs and T17b EPCs stimulate the production of proangiogenic molecules, we additionally performed an MMP3 ELISA as well as an NO assay. It is well known that angiogenesis is a well-orchestrated process involving a multitude of sequentially coordinated events. One important step is the degradation of the extracellular matrix in order to promote endothelial cell invasion. In this context, the MMPs play an important role through degrading the extracellular matrix [Chang and Werb, 2001; Chen et al., 2013]. As MMP-3 is discussed in the literature as an important protease in angiogenesis [Sage et al., 2003; Jin et al., 2006], we measured the production of MMP-3 in the 2 -dimensional cell culture. In this context, we could detect the highest amount of MMP- 3 in the coculture group containing 75\% MSCs. Interestingly, CM from MSCs reduced the amount of MMP-3 in T17b EPCs (T17b EPC $\mathrm{CM}$ ), suggesting that the stimulation of MMP-3 production is dependent on heterotypic cell contacts between MSCs and T17b EPCs, and not paracrine-acting mediators. It has been shown that NO is an important signal molecule regulating angiogenesis as well as vascular to-
224

Cells Tissues Organs 2017;204:218-227 DOI: $10.1159 / 000478654$
Steiner/Köhn/Beier/Stürzl/Horch/ Arkudas 
nicity and thereby the tissue perfusion [Fukumura et al., 2006; Isenberg et al., 2009]. In our experiments, we could detect a constant NO production in all groups without any differences. These results, especially regarding the generation of bioartificial tissues based on T17b EPCs and MSCs, are very promising. Although still speculative, the coimplantation of both cell types could support vascularization through the secretion of VEGF, facilitated sprouting, as well as endothelial cell invasion by MMP-3 release and enhanced perfusion of the surrounding tissue by NO.

Knowing that endothelial cells and EPCs can induce the osteogenic differentiation of MSCs [Thébaud et al., 2012; Fu et al., 2015], we measured the early osteogenic differentiation marker ALP as well as the concentration of the osteogenic growth factor BMP-2. In the pertinent literature, ALP is described as an early osteogenic differentiation marker. In this regard, Hager et al. [2009] could demonstrate that the osteogenic differentiation of osteoblasts was stimulated by coculturing with endothelial cells $48 \mathrm{~h}$ after cell seeding. Interestingly, we could detect a statistically significant downregulation of ALP activity in the coculture group. BMP-2 is a well-known osteogenic growth factor that supports osteogenic differentiation as well as bone formation [Betz et al. 2010; Hosogane et al., 2010]. However, concordantly with the results from the ALP assay, the BMP-2 levels have been reduced in the coculture groups suggesting that T17b EPCs inhibit the osteogenic differentiation of MSCs. These results are surprising given the fact that the coimplantation of MSCs and EPCs leads to enhanced vascularization and bone formation in vivo [Usami et al., 2009; Zigdon-Giladi et al., 2015]. Taking into consideration that we chose an EPC cell line, these results might be partially explained. Although described in the literature, the 48- and 72-h time points might be too early for measuring the osteogenic differentiation in our coculture system [Hager et al.,
2009]. Furthermore, it could be possible that T17b EPCs induce the differentiation of MSCs into another cell type. In this context, Goerke et al. [2012] demonstrated that MSCs can be differentiated into smooth muscle cells upon cocultivation with EPCs.

\section{Conclusion}

Our data reveal that T17b EPCs increase the proliferation rate of MSCs. Furthermore, MSCs reduced the apoptosis of T17b EPCs. These effects seemed to be regulated through heterotypic cell contacts and paracrine-acting factors. In this context, it might be intriguing to investigate whether VEGF secreted from MSCs is the antiapoptotic reagent. As we could prove that T17b EPC/MSC cocultures also produce angiogenic molecules, this coculture system is a promising tool for further in vivo experiments. Taking into account that T17b EPCs inhibited the osteogenic differentiation of MSCs, further studies are required. Our study shows that the ideal coculture contains 25\% T17b EPCs and 75\% MSCs. This cell ratio displayed a robust proliferation rate, the highest VEGF and MMP-3 secretion, as well as the lowest apoptosis rate.

\section{Acknowledgements}

This study was funded by the ELAN-Fonds (14-08-22-1-Steiner) of the University of Erlangen-Nürnberg and by the Xue Hong and Hans Georg Geis Stiftung. We thank Antonis K. Hatzopoulos (Vanderbilt University School of Medicine) for kindly providing T17b cells. We thank Alex Haydl for revising the manuscript.

\section{Disclosure Statement}

The authors declare no conflicts of interest.

\section{References}

Ahn, G.O., J.M. Brown (2009) Role of endothelial progenitors and other bone marrow-derived cells in the development of the tumor vasculature. Angiogenesis 12: 159-164.

Arkudas, A., J. Tjiawi, O. Bleiziffer, L. Grabinger, E. Polykandriotis, J.P. Beier, M. Sturzl, R.E. Horch, U. Kneser (2007) Fibrin gel-immobilized VEGF and bFGF efficiently stimulate angiogenesis in the AV loop model. Mol Med 13: $480-487$.

Asahara, T., H. Masuda, T. Takahashi, C. Kalka, C. Pastore, M. Silver, M. Kearne, M. Magner,

Antiapoptotic and Proangiogenic Effects of Cocultivation of MSCs and EPCs
J.M. Isner (1999) Bone marrow origin of endothelial progenitor cells responsible for postnatal vasculogenesis in physiological and pathological neovascularization. Circ Res 85 : 221-228.

Asahara, T., T. Murohara, A. Sullivan, M. Silver, R. van der Zee, T. Li, B. Witzenbichler, G. Schatteman and J.M. Isner (1997) Isolation of putative progenitor endothelial cells for angiogenesis. Science 275: 964-967.

Betz, O.B., V.M. Betz, A. Abdulazim, R. Penzkofer, B. Schmitt, C. Schroder, S. Mayer-Wagner, P.
Augat, V. Jansson, P.E. Muller (2010) The repair of critical-sized bone defects using expedited, autologous BMP-2 gene-activated fat implants. Tissue Eng Part A 16: 1093-1101.

Bleiziffer, O., M. Hammon, E. Naschberger, K Lipnik, A. Arkudas, S. Rath, G. Pryymachuk, J.P. Beier, M. Sturzl, R.E. Horch, U. Kneser (2011) Endothelial progenitor cells are integrated in newly formed capillaries and alter adjacent fibrovascular tissue after subcutaneous implantation in a fibrin matrix. J Cell Mol Med 15: 2452-2461. 
Bleiziffer, O., R.E. Horch, M. Hammon, A. Arkudas, E. Naschberger, S. Rath, G. Pryymachuk, J.P. Beier, A.K. Hatzopoulos, M. Sturzl, U. Kneser (2009) T17b murine embryonal endothelial progenitor cells can be induced towards both proliferation and differentiation in a fibrin matrix. J Cell Mol Med 13: 926-935.

Brandl, A., Q. Yuan, A.M. Boos, J.P. Beier, A. Arkudas, U. Kneser, R.E. Horch, O. Bleiziffer (2014) A novel early precursor cell population from rat bone marrow promotes angiogenesis in vitro. BMC Cell Biol 15: 12.

Chang, C., Z. Werb (2001) The many faces of metalloproteases: cell growth, invasion, angiogenesis and metastasis. Trends Cell Biol 11: S37-S43.

Chen, Q., M. Jin, F. Yang, J. Zhu, Q. Xiao, L. Zhang (2013) Matrix metalloproteinases: inflammatory regulators of cell behaviors in vascular formation and remodeling. Mediators Inflamm 2013: 928315.

Folkman, J. (1998) Therapeutic angiogenesis in ischemic limbs. Circulation 97: 1108-1110.

Fu, W.L., Z. Xiang, F.G. Huang, Z.P. Gu, X.X. Yu, S.Q. Cen, G. Zhong, X. Duan, M. Liu (2015) Coculture of peripheral blood-derived mesenchymal stem cells and endothelial progenitor cells on strontium-doped calcium polyphosphate scaffolds to generate vascularized engineered bone. Tissue Eng Part A 21: 948-959.

Fukumura, D., S. Kashiwagi, R.K. Jain (2006) The role of nitric oxide in tumour progression. Nat Rev Cancer 6: 521-534.

Gerber, H.P., V. Dixit, N. Ferrara (1998a) Vascular endothelial growth factor induces expression of the antiapoptotic proteins $\mathrm{Bcl}-2$ and A1 in vascular endothelial cells. J Biol Chem 273: 13313-13316.

Gerber, H.P., A. McMurtrey, J. Kowalski, M. Yan, B.A. Keyt, V. Dixit, N. Ferrara (1998b) Vascular endothelial growth factor regulates endothelial cell survival through the phosphatidylinositol 3'-kinase/Akt signal transduction pathway: requirement for Flk-1/KDR activation. J Biol Chem 273: 30336-30343.

Goerke, S.M., J. Plaha, S. Hager, S. Strassburg, N. Torio-Padron, G.B. Stark, G. Finkenzeller (2012) Human endothelial progenitor cells induce extracellular signal-regulated kinasedependent differentiation of mesenchymal stem cells into smooth muscle cells upon cocultivation. Tissue Eng Part A 18: 2395-2405.

Grellier, M., N. Ferreira-Tojais, C. Bourget, R. Bareille, F. Guillemot, J. Amedee (2009) Role of vascular endothelial growth factor in the communication between human osteoprogenitors and endothelial cells. J Cell Biochem 106: 390-398.

Hager, S., F.M. Lampert, H. Orimo, G.B. Stark, G. Finkenzeller (2009) Up-regulation of alkaline phosphatase expression in human primary osteoblasts by cocultivation with primary endothelial cells is mediated by p 38 mitogen-activated protein kinase-dependent mRNA stabilization. Tissue Eng Part A 15: 3437-3447.
Hatzopoulos, A.K., J. Folkman, E. Vasile, G.K. Eiselen, R.D. Rosenberg (1998) Isolation and characterization of endothelial progenitor cells from mouse embryos. Development 125: 1457-1468.

Horch, R.E., J.P. Beier, U. Kneser, A. Arkudas (2014) Successful human long-term application of in situ bone tissue engineering. J Cell Mol Med 18: 1478-1485.

Hosogane, N., Z. Huang, B.A. Rawlins, X. Liu, O. Boachie-Adjei, A.L. Boskey, W. Zhu (2010) Stromal derived factor-1 regulates bone morphogenetic protein 2-induced osteogenic differentiation of primary mesenchymal stem cells. Int J Biochem Cell Biol 42: 1132-1141.

Iba, O., H. Matsubara, Y. Nozawa, S. Fujiyama, K. Amano, Y. Mori, H. Kojima, T. Iwasaka (2002) Angiogenesis by implantation of peripheral blood mononuclear cells and platelets into ischemic limbs. Circulation 106: 2019-2025.

Isenberg, J.S., G. Martin-Manso, J.B. Maxhimer, D.D. Roberts (2009) Regulation of nitric oxide signalling by thrombospondin 1: implications for anti-angiogenic therapies. Nat Rev Cancer 9: 182-194.

Janeczek Portalska, K., A. Leferink, N. Groen, H. Fernandes, L. Moroni, C. van Blitterswijk, J. de Boer (2012) Endothelial differentiation of mesenchymal stromal cells. PLoS One 7: e46842.

Jin, X., M. Yagi, N. Akiyama, T. Hirosaki, S. Higashi, C.Y. Lin, R.B. Dickson, H. Kitamura, K. Miyazaki (2006) Matriptase activates stromelysin (MMP-3) and promotes tumor growth and angiogenesis. Cancer Sci 97: 1327-1334.

Ko, H.C., B.K. Milthorpe, C.D. McFarland (2007) Engineering thick tissues - the vascularisation problem. Eur Cell Mater 14: 1-18, discussion 18-19.

Kocher, A.A., M.D. Schuster, M.J. Szabolcs, S. Takuma, D. Burkhoff, J. Wang, S. Homma, N.M. Edwards, S. Itescu (2001) Neovascularization of ischemic myocardium by human bonemarrow-derived angioblasts prevents cardiomyocyte apoptosis, reduces remodeling and improves cardiac function. Nat Med 7: 430436.

Kolbe, M., Z. Xiang, E. Dohle, M. Tonak, C.J. Kirkpatrick, S. Fuchs (2011) Paracrine effects influenced by cell culture medium and consequences on microvessel-like structures in cocultures of mesenchymal stem cells and outgrowth endothelial cells. Tissue Eng Part A 17: 2199-2212.

Koob, S., N. Torio-Padron, G.B. Stark, C. Hannig, Z. Stankovic, G. Finkenzeller (2011) Bone formation and neovascularization mediated by mesenchymal stem cells and endothelial cells in critical-sized calvarial defects. Tissue Eng Part A 17: 311-321.
Kupatt, C., R. Hinkel, M. Lamparter, M.L. von Bruhl, T. Pohl, J. Horstkotte, H. Beck, S. Muller, S. Delker, F.J. Gildehaus, H. Buning, A.K. Hatzopoulos, P. Boekstegers (2005a) Retroinfusion of embryonic endothelial progenitor cells attenuates ischemia-reperfusion injury in pigs: role of phosphatidylinositol 3-kinase/AKT kinase. Circulation 112(9 Suppl): I117-I122.

Kupatt, C., J. Horstkotte, G.A. Vlastos, A. Pfosser, C. Lebherz, M. Semisch, M. Thalgott, K. Buttner, C. Browarzyk, J. Mages, R. Hoffmann, A. Deten, M. Lamparter, F. Muller, H. Beck, H. Buning, P. Boekstegers, A.K. Hatzopoulos (2005b) Embryonic endothelial progenitor cells expressing a broad range of proangiogenic and remodeling factors enhance vascularization and tissue recovery in acute and chronic ischemia. FASEB J 19: 15761578.

Moimas, S., B. Manasseri, G. Cuccia, F. Stagno d'Alcontres, S. Geuna, L. Pattarini, L. Zentilin, M. Giacca, M.R. Colonna (2015) AAV vector encoding human VEGF165-transduced pectineus muscular flaps increase the formation of new tissue through induction of angiogenesis in an in vivo chamber for tissue engineering: a technique to enhance tissue and vessels in microsurgically engineered tissue. J Tissue Eng 6: 2041731415611717.

Rottensteiner, U., B. Sarker, D. Heusinger, D. Dafinova, S.N. Rath, J.P. Beier, U. Kneser, R.E. Horch, R. Detsch, A.R. Boccaccini, A. Arkudas (2014) In vitro and in vivo biocompatibility of alginate dialdehyde/gelatin hydrogels with and without nanoscaled bioactive glass for bone tissue engineering applications. Materials 7: 1957-1974.

Rouwkema, J., A. Khademhosseini (2016) Vascularization and angiogenesis in tissue engineering: beyond creating static networks. Trends Biotechnol 34: 733-745.

Sage, E.H., M. Reed, S.E. Funk, T. Truong, M. Steadele, P. Puolakkainen, D.H. Maurice, J.A. Bassuk (2003) Cleavage of the matricellular protein SPARC by matrix metalloproteinase 3 produces polypeptides that influence angiogenesis. J Biol Chem 278: 37849-37857.

Steiner, D., F. Lampert, G.B. Stark, G. Finkenzeller (2012) Effects of endothelial cells on proliferation and survival of human mesenchymal stem cells and primary osteoblasts. J Orthop Res 30: 1682-1689.

Street, J., M. Bao, L. deGuzman, S. Bunting, F.V. Peale, Jr., N. Ferrara, H. Steinmetz, J. Hoeffel, J.L. Cleland, A. Daugherty, N. van Bruggen, H.P. Redmond, R.A. Carano, E.H. Filvaroff (2002) Vascular endothelial growth factor stimulates bone repair by promoting angiogenesis and bone turnover. Proc Natl Acad Sci USA 99: 9656-9661.

Street, J., B. Lenehan (2009) Vascular endothelial growth factor regulates osteoblast survival evidence for an autocrine feedback mechanism. J Orthop Surg Res 4: 19. 
Thébaud, N.B., R. Siadous, R. Bareille, M. Remy, R. Daculsi, J. Amedee, L. Bordenave (2012) Whatever their differentiation status, human progenitor derived - or mature - endothelial cells induce osteoblastic differentiation of bone marrow stromal cells. J Tissue Eng Regen Med 6: e51-e60.

Usami, K., H. Mizuno, K. Okada, Y. Narita, M. Aoki, T. Kondo, D. Mizuno, J. Mase, H. Nishiguchi, H. Kagami, M. Ueda (2009) Composite implantation of mesenchymal stem cells with endothelial progenitor cells enhances tissueengineered bone formation. J Biomed Mater Res A 90: 730-741.

Vielreicher, M., M. Gellner, U. Rottensteiner, R.E. Horch, A. Arkudas, O. Friedrich (2015) Multiphoton microscopy analysis of extracellular collagen I network formation by mesenchymal stem cells. J Tissue Eng Regen Med, Epub ahead of print.

Villars, F., L. Bordenave, R. Bareille, J. Amedee (2000) Effect of human endothelial cells on human bone marrow stromal cell phenotype: role of VEGF? J Cell Biochem 79: 672-685.
Villars, F., B. Guillotin, T. Amedee, S. Dutoya, L. Bordenave, R. Bareille, J. Amedee (2002) Effect of HUVEC on human osteoprogenitor cell differentiation needs heterotypic gap junction communication. Am J Physiol Cell Physiol 282: C775-C785.

Wang, S., Z. Chen, X. Tang, H. Liu, L. Yang, Y. Wang (2015) Transplantation of vascular endothelial growth factor 165 -transfected endothelial progenitor cells for the treatment of limb ischemia. Mol Med Rep 12: 4967-4974.

Weigand, A., J.P. Beier, A. Hess, T. Gerber, A. Arkudas, R.E. Horch, A.M. Boos (2015) Acceleration of vascularized bone tissue-engineered constructs in a large animal model combining intrinsic and extrinsic vascularization. Tissue Eng Part A 21: 1680-1694.
Wen, L., Y. Wang, N. Wen, G. Yuan, M. Wen, L. Zhang, Q. Liu, Y. Liang, C. Cai, X. Chen Y. Ding (2016) Role of endothelial progenitor cells in maintaining stemness and enhancing differentiation of mesenchymal stem cells by indirect cell-cell interaction. Stem Cells Dev 25: $123-138$.

Yoder, M.C., L.E. Mead, D. Prater, T.R. Krier, K.N. Mroueh, F. Li, R. Krasich, C.J. Temm, J.T. Prchal, D.A. Ingram (2007) Redefining endothelial progenitor cells via clonal analysis and hematopoietic stem/progenitor cell principals. Blood 109: 1801-1809.

Young, P.P., D.E. Vaughan, A.K. Hatzopoulos (2007) Biologic properties of endothelial progenitor cells and their potential for cell therapy. Prog Cardiovasc Dis 49: 421-429.

Zigdon-Giladi, H., T. Bick, D. Lewinson, E.E. Machtei (2015) Co-transplantation of endothelial progenitor cells and mesenchymal stem cells promote neovascularization and bone regeneration. Clin Implant Dent Relat Res 17: 353-359. 